1 Hacettepe Journal of Mathematics and Statistics

$\bigcap$ Volume $44(5)$ (2015), $1087-1097$

\title{
Inversion Laplace transform for integrodifferential parabolic equation with purely nonlocal conditions
}

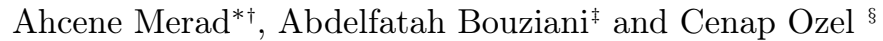

\begin{abstract}
In this paper we prove the existence, uniqueness, and continuous dependence upon the data of solution to integrodifferential parabolic equation with purely nonlocal integral conditions. The proofs are based on a priori estimates and Laplace transform method. Finally, we obtain a solution using a numerical technique which is called Stehfest algorithm by inverting the Laplace transform.
\end{abstract}

Keywords: Integrodifferential parabolic equation, Approximate solution, Nonlocal purely integral conditions, Stehfest Algorithm.

2000 AMS Classification: 44A10, 34A12,35L10

Received 07/03/2012 : Accepted 09/09/2014 Doi : 10.15672/HJMS.2015449667

\section{Introduction}

In this paper we are concerned with the following parabolic integrodifferential equation

$$
\frac{\partial v}{\partial t}(x, t)-\frac{\partial^{2} v}{\partial x^{2}}(x, t)=\int_{0}^{t} a(t-s) v(x, s) d s, 0<x<1,0<t \leq T,
$$

subject to the initial condition

$$
v(x, 0)=\Phi(x), 0<x<1
$$

*Department of Mathematics, Larbi Ben M'hidi University, 04000, Algeria, and Department of Mathematics, University of Jijel, 18000 Jijel, Algeria , Email: merad_ahcene@yahoo.fr

${ }^{\dagger}$ Corresponding Author.

${ }^{\ddagger}$ Department of Mathematics, Larbi Ben M’hidi University,04000, Algeria , Email: author.two@mail.com

${ }^{\S}$ Department of Mathematics, Dokuz Eylul University, Izmir, Turkey, Email: cenap.ozel@gmail.com 
and the purely nonlocal (integral) conditions

$$
\begin{aligned}
& \int_{0}^{1} v(x, t) d x=r(t), 0<t \leq T, \\
& \int_{0}^{1} x v(x, t) d x=q(t), 0<t \leq T,
\end{aligned}
$$

where $v$ is an unknown function, $r, q$, and $\Phi(x)$ are given functions supposed to be sufficiently regular, $a$ is suitably defined function satisfying certain some conditions that will be specified later and $T$ is a positive constant number.

Some problems from modern physics and science can be described in terms of partial differential equations with nonlocal conditions. For instance, the nonlocal term of our problem ( i.e $\int_{0}^{t} a(t-s) v(x, s) d s$ ) appears in the modeling of the quasi-static flexure of a thermo-elastic rod [10, 12]. First this problem with the more general second-order parabolic equation or a $2 m$-parabolic equation has been studied by the second author using the energy-integral methods and the Rothe method in $[10,12,14]$ and [28] respectively. For other models we refer to [7, 12, 13, 15], [16]-[19],[20]-[27], [29]-[34]. The problem (1.1) - (1.3) is studied by using the Rothe method in [21]. On the other hand Ang in [2] considered a one-dimensional heat equation with nonlocal integral conditions and applied the Laplace transform to the problem. Then he used some numerical techniques to obtain a numerical solution of the inverse Laplace transform.

Recently the various types of the partial differential equations with nonlocal conditions have been studied by [3], [4] and [5], [6] and [8], [9].

This paper is organized as follows. In Section 2, we introduce some certain function spaces what we need in this work, and also give a reduction of our problem to another equivalent problem with the homogeneous integral conditions. In Section 3, we establish the existence of the solution by the Laplace transform method. In Section 4, we deal with a priori estimate which gives the uniqueness and continuous dependence upon the given data.

\section{Statement of the Problem and Notations}

Since integral conditions are not homogenous, it is convenient to convert the problem $(1.1)-(1.3)$ to an equivalent problem with the homogenous integral conditions. For this reason, we introduce a new function $u(x, t)$ representing the deviation of the function $v(x, t)$ as

$$
u(x, t)=v(x, t)-w(x, t), 0<x<1,0<t \leq T,
$$

where

$$
w(x, t)=6(2 q(t)-r(t)) x-2(3 q(t)-2 r(t)) .
$$

The problem (1.1) - (1.3) with non-homogenous integral conditions (1.3) can be equivalently reduced to the problem of finding a function $u$ satisfying

$$
\begin{aligned}
& \frac{\partial u}{\partial t}(x, t)-\frac{\partial^{2} u}{\partial x^{2}}(x, t)=\int_{0}^{t} a(t-s) u(x, s) d s, 0<x<1,0<t \leq T, \\
& u(x, 0)=\varphi(x), 0<x<1,
\end{aligned}
$$




$$
\begin{aligned}
& \int_{0}^{1} u(x, t) d x=0,0<t \leq T, \\
& \int_{0}^{1} x u(x, t) d x=0,0<t \leq T,
\end{aligned}
$$

where

$$
\varphi(x)=\Phi(x)-w(x, 0) .
$$

The solution of problem (1.1) - (1.3) will be obtained by the relation (2.1) and (2.2). Let $H$ be the Hilbert space with the norm $\|\cdot\|_{H}$ and $L^{2}(0,1)$ be the space of all the square integrable functions on the interval $(0,1)$. Now we are ready to introduce some appropriate function spaces what we need in this work.

2.1. Definition. (i) We denote by $L^{2}(0, T ; H)$ the set of all measurable functions $u(., t)$ from $(0, T)$ into $H$ equipped with the norm

$$
\|u\|_{L^{2}(0, T ; H)}=\left(\int_{0}^{T}\|u(., t)\|_{H}^{2} d t\right)^{1 / 2}<\infty .
$$

(ii) The space $C(0, T ; H)$ is the set of all continuous functions $u(., t):(0, T) \longrightarrow H$ equipped with the norm

$$
\|u\|_{C(0, T ; H)}=\max _{0 \leq t \leq T}\|u(., t)\|_{H}<\infty .
$$

We denote by $C_{0}(0,1)$ the space of all continuous functions with a compact support in $(0,1)$. Since such functions are Lebesgue integrable with respect to $x$, we can define a bilinear form on $C_{0}(0,1)$ given by

$$
(u, w)=\int_{0}^{1} J_{x}^{m} u . J_{x}^{m} w d x, m \geq 1,
$$

where

$$
J_{x}^{m} u=\int_{0}^{x} \frac{(x-\zeta)^{m-1}}{(m-1) !} u(\zeta, t) d \zeta ; \text { for } m \geq 1 .
$$

We know that the bilinear form $(2.6)$ is a scalar product on $C_{0}(0,1)$ but $C_{0}(0,1)$ is not a complete space.

2.2. Definition. Denote by $B_{2}^{m}(0,1)$, the completion of $C_{0}(0,1)$ for the scalar product (2.6), which is denoted by $(.,)_{B_{2}^{m}(0,1)}$, introduced in [11]. By the norm of a function $u$ from $B_{2}^{m}(0,1), m \geq 1$, we understand the nonnegative number:

$$
\|u\|_{B_{2}^{m}(0,1)}=\left(\int_{0}^{1}\left(J_{x}^{m} u\right)^{2} d x\right)^{1 / 2}=\left\|J_{x}^{m} u\right\|, \text { for } m \geq 1 .
$$

From [11] we have the following lemma.

2.3. Lemma. For all $m \in \mathbb{Z}^{+}$the following inequality

$$
\|u\|_{B_{2}^{m}(0,1)}^{2} \leq \frac{1}{2}\|u\|_{B_{2}^{m-1}(0,1)}^{2}
$$

holds. 
2.4. Corollary. For all $m \in \mathbb{Z}^{+}$we have the elementary inequality

$$
\|u\|_{B_{2}^{m}(0,1)}^{2} \leq\left(\frac{1}{2}\right)^{m}\|u\|_{L^{2}(0,1)}^{2} .
$$

2.5. Definition. We denote by $L^{2}\left(0, T ; B_{2}^{m}(0,1)\right)$ the space of functions which are square integrable in the Bochner sense with the scalar product

$$
(u, w)_{L^{2}\left(0, T ; B_{2}^{m}(0,1)\right)}=\int_{0}^{T}(u(., t), w(., t))_{B_{2}^{m}(0,1)} d t .
$$

Since the space $B_{2}^{m}(0,1)$ is a Hilbert space, it can be shown that $L^{2}\left(0, T ; B_{2}^{m}(0,1)\right)$ is also a Hilbert space. The set of all continuous functions in $[0, T]$ equipped with the norm

$$
\sup _{0 \leq t \leq T}\|u(., t)\|_{B_{2}^{m}(0,1)}
$$

will be denoted by $C\left(0, T ; B_{2}^{m}(0,1)\right)$.

2.6. Corollary. The following imbedding $L^{2}(0,1) \longrightarrow B_{2}^{m}(0,1)$ is continuous for $m \geq 1$.

By Lemma 1.3.19 in [25], we have the following result.

2.7. Lemma (Gronwall Lemma). Let $f_{1}(t), f_{2}(t) \geq 0$ be two integrable functions on $[0, T]$, let us suppose that $f_{2}(t)$ is nondecreasing. If we have

$$
f_{1}(\tau) \leq f_{2}(\tau)+c \int_{0}^{\tau} f_{1}(t) d t, \forall \tau \in[0, T]
$$

where $c \in \mathbb{R}^{+}$then we have

$$
f_{1}(t) \leq f_{2}(t) \exp (c t), \forall t \in[0, T] .
$$

\section{Existence of the Solution.}

The Laplace transform method is an efficient way to solve many ordinary and partial differential equations. But the main difficulty with the Laplace transform method is in the inverting the Laplace domain solution into the real domain. In this section we will carry out the Laplace transform techniques to find solutions of the partial differential equations.

Suppose that $v(x, t)$ is defined and is of exponential order for $t \geq 0$ i.e. there exists $A$, $\gamma>0$ and $t_{0}>0$ such that $|v(x, t)| \leq A \exp (\gamma t)$ for $t \geq t_{0}$. Then the Laplace transform $V(x, s)$ exists and it is given by

$$
V(x, s)=\{v(x, t) ; t \longrightarrow s\}=\int_{0}^{\infty} v(x, t) \exp (-s t) d t,
$$

where $s$ is a positive real parameter. Applying the Laplace transform on both sides of (1.1), we have

$$
(s-A(s)) V(x, s)-\frac{d^{2}}{d x^{2}} V(x, s)=s \Phi(x),
$$

where $G(x, s)=\{g(x, t) ; t \longrightarrow s\}$. Similarly, we have

$$
\begin{aligned}
\int_{0}^{1} V(x, s) d x & =R(s), \\
\int_{0}^{1} x V(x, s) d x & =Q(s),
\end{aligned}
$$


where

$$
\begin{aligned}
& R(s)=\{r(t) ; t \longrightarrow s\}, \\
& Q(s)=\{q(t) ; t \longrightarrow s\} .
\end{aligned}
$$

Now we have three distinguished cases:

Case 1. $s-A(s)>0$.

Case 2. $s-A(s)<0$.

Case 3. $s-A(s)=0$.

Here we consider only Case 2 and 3, because Case 1 can be dealt as like in [2]. For $(s-A(s))=0$, we have

$$
\frac{d^{2}}{d x^{2}} V(x, s)=-s \Phi(x) .
$$

The general solution for Case 3 is given by

$$
V(x, s)=-\int_{0}^{x} \int_{0}^{y}[s \Phi(x)] d z d y+C_{1}(s) x+C_{2}(s) .
$$

Putting the integral conditions (3.3) in (3.5) we get

$$
\begin{aligned}
& \frac{1}{2} C_{1}(s)+C_{2}(s) \\
= & \int_{0}^{1} \int_{0}^{x} \int_{0}^{y}[s \Phi(x)] d z d y+R(s), \\
& \frac{1}{3} C_{1}(s)+\frac{1}{2} C_{2}(s) \\
= & \int_{0}^{1} \int_{0}^{x} \int_{0}^{y} x[s \Phi(x)] d z d y+Q(s),
\end{aligned}
$$

and

$$
\begin{aligned}
C_{1}(s)= & 12 \int_{0}^{1} \int_{0}^{x} \int_{0}^{y} x[s \Phi(x)] d z d y- \\
& 6 \int_{0}^{1} \int_{0}^{x} \int_{0}^{y}[s \Phi(x)] d z d y+ \\
& 12 Q(s)-6 R(s), \\
C_{2}(s)= & 4 \int_{0}^{1} \int_{0}^{x} \int_{0}^{y}[s \Phi(x)] d z d y- \\
& 6 \int_{0}^{1} \int_{0}^{x} \int_{0}^{y} x[s \Phi(x)] d z d y- \\
& 6 Q(s)+4 R(s) .
\end{aligned}
$$

For Case 2, that is, when $(s-A(s))<0$, using the method of variation of parameters, we have the general solution as

$$
\begin{aligned}
V(x, s)= & \frac{1}{\sqrt{A(s)-s^{2}}} \int_{0}^{x}(s \Phi(x)) . \\
& \sin (\sqrt{A(s)-s})(x-\tau) d \tau+d_{1}(s) \cos \sqrt{(A(s)-s)} x+ \\
& d_{2}(s) \sin \sqrt{(A(s)-s)} x .
\end{aligned}
$$


From the integral conditions (3.3) we get

$$
\begin{aligned}
& d_{1}(s) \int_{0}^{1} \cos \sqrt{(A(s)-s)} x d x+d_{2}(s) \int_{0}^{1} \sin \sqrt{(A(s)-s)} x d x \\
= & R(s)-\frac{1}{\sqrt{A(s)-s^{2}}} \int_{0}^{1} \int_{0}^{x}(s \Phi(x)) \cdot \\
& \sin (\sqrt{A(s)-s})(x-\tau) d \tau d x, \\
& d_{1}(s) \int_{0}^{1} x \cos \sqrt{(A(s)-s)} x d x+d_{2}(s) \int_{0}^{1} x \sin \sqrt{(A(s)-s)} x d x \\
= & Q(s)-\frac{1}{\sqrt{A(s)-s}} \int_{0}^{1} \int_{0}^{x} x(s \Phi(x)) \cdot \\
& \sin (\sqrt{A(s)-s})(x-\tau) d \tau d x .
\end{aligned}
$$

Thus $d_{1}, d_{2}$ are given by

$$
\left(\begin{array}{l}
d_{1}(s) \\
d_{2}(s)
\end{array}\right)=\left(\begin{array}{ll}
a_{11}(s) & a_{12}(s) \\
a_{21}(s) & a_{22}(s)
\end{array}\right)^{-1} \cdot\left(\begin{array}{l}
b_{1}(s) \\
b_{2}(s)
\end{array}\right)
$$

where

$$
\begin{aligned}
a_{11}(s)= & \int_{0}^{1} \cos \sqrt{(A(s)-s)} x d x, \\
a_{12}(s)= & \int_{0}^{1} \sin \sqrt{(A(s)-s)} x d x, \\
a_{21}(s)= & \int_{0}^{1} x \cos \sqrt{(A(s)-s)} x d x, \\
a_{22}(s)= & \int_{0}^{1} x \sin \sqrt{(A(s)-s)} x d x, \\
b_{1}(s)= & R(s)-\frac{1}{\sqrt{A(s)-s}} \int_{0}^{1} \int_{0}^{x}(s \Phi(x)) . \\
b_{2}(s)= & \sin \left(\sqrt{A(s)-s)(x-\tau) d \tau d x,} \frac{1}{\sqrt{A(s)-s}} \int_{0}^{1} \int_{0}^{x} x(s \Phi(x)) .\right. \\
& \sin (\sqrt{A(s)-s)(x-\tau) d \tau d x .}
\end{aligned}
$$

If it is not possible to calculate the integrals directly, then we can calculate them numerically. So we can approximate them similarly as done in [2]. If the Laplace inversion is possibly computed directly for (3.5) and (3.8), then we reach the solution explicitly. Otherwise we have to use the suitable approximate technique to get numerical solution, therefore we need the numerical inversion of the Laplace transform. Considering $A(s)-$ $s=k(s)$ and using Gauss's formula given in [1] we have the following appoximations of 
the integrals

$$
\begin{aligned}
& \int_{0}^{1}\left(\begin{array}{l}
1 \\
x
\end{array}\right) \cos \sqrt{k(s)} x d x \\
\simeq & \frac{1}{2} \sum_{i=1}^{N} w_{i}\left(\begin{array}{c}
1 \\
\frac{1}{2}\left[x_{i}+1\right]
\end{array}\right) \cos \left(\sqrt{k(s)} \frac{1}{2}\left[x_{i}+1\right]\right) \\
& \int_{0}^{1}\left(\begin{array}{l}
1 \\
x
\end{array}\right) \sin \sqrt{k(s)} x d x \\
\simeq & \frac{1}{2} \sum_{i=1}^{N} w_{i}\left(\begin{array}{c}
1 \\
\frac{1}{2}\left[x_{i}+1\right]
\end{array}\right) \sin \left(\sqrt{k(s)} \frac{1}{2}\left[x_{i}+1\right]\right) \\
& \int_{0}^{x}(s \Phi(x)) \sin (\sqrt{k(s)})(x-\tau) d \tau \\
& \frac{x}{2} \sum_{i=1}^{N} w_{i}\left[s \Phi\left(\frac{x}{2}\left[x_{i}+1\right]\right)\right] \\
& \sin \left(\sqrt{k(s)}\left[x-\frac{x}{2}\left[x_{i}+1\right]\right]\right) \\
& \int_{0}^{1}\left[[s \Phi(\tau)] \int_{\tau}^{1}\left(\begin{array}{l}
1 \\
x
\end{array}\right) \sin (\sqrt{k(s)})(x-\tau) d x\right] d \tau \\
\simeq & \frac{1}{2} \sum_{i=1}^{N} w_{i}\left[s \Phi\left(\frac{1}{2}\left[x_{i}+1\right]\right)\right] \\
& \left(\frac{1-\frac{1}{2}\left[x_{i}+1\right]}{2}\right) \sum_{i=1}^{N} w_{j}\left(\frac{1-\frac{1}{2}\left[x_{i}+1\right]}{2} x_{j}+\frac{1-\frac{1}{2}\left[x_{i}+1\right]}{2}\right) \\
& \sin \left(\sqrt{k(s)}\left[\frac{1-\frac{1}{2}\left[x_{i}+1\right]}{2} x_{j}+\frac{1+\frac{1}{2}\left[x_{i}+1\right]}{2}-\frac{1}{2}\left(x_{i}+1\right)\right]\right),
\end{aligned}
$$

where $x_{i}$ and $w_{i}$ are the abscissa and weights defined as

$$
x_{i}: i^{t h} \text { zero of } P_{n}(x), \omega_{i}=2 /\left(1-x_{i}^{2}\right)\left[P_{n}^{\prime}(x)\right]^{2} \text {. }
$$

Their tabulated values can be found in [1] for different values of $N$.

3.1. A numerical inversion of a Laplace transform. Sometimes an analytical inversion of the Laplace domain solution is difficult to obtain. Therefore, a numerical inversion method has to be required. An important comparison of four frequently used numerical Laplace inversion algorithms is given by H. Hassanzadeh et al in [24]. Here we use the Stehfest algorithm [34] that is easy to implement. This numerical technique was first introduced by Graver [22] and then its algorithm is improved by [34]. The Stehfest algorithm approximates the time domain solution as

$$
v(x, t) \approx \frac{\ln 2}{t} \sum_{n=1}^{2 m} \beta_{n} V\left(x ; \frac{n \ln 2}{t}\right),
$$

where $m$ is a positive integer,

$$
\beta_{n}=(-1)^{n+m} \sum_{k=\left[\frac{n+1}{2}\right]}^{\min (n, m)} \frac{k^{m}(2 k) !}{(m-k) ! k !(k-1) !(n-k) !(2 k-n) !},
$$

and $[q]$ is the integer part of the real number $q$. 


\section{A Numerical Example}

In this section we perform some results of numerical computations using the Laplace transform method proposed in the previous section. This technique can be carried out to solve the problem defined by the problem (1.1) - (1.3). The method is easily applicable via Matlab 7.9.3 program. So we can give the following example.

4.1. Example. We take the integrodifferential equation

$$
\begin{aligned}
\frac{\partial v}{\partial t}(x, t)-\frac{\partial^{2} v}{\partial x^{2}}(x, t) & =\int_{0}^{t} \exp (t-s) u(x, s) d s, 0<x<1,0<t \leq T, \\
v(x, 0) & =\sin x, 0<x<1, \\
\int_{0}^{1} v(x, t) d x & =0,0<t \leq T, \\
\int_{0}^{1} x v(x, t) d x & =0,0<t \leq T .
\end{aligned}
$$

In this case the exact solution is given by

$$
v(x, t)=\exp (-t) \cdot \cos t \cdot \sin x, 0<x<1,0<t \leq T .
$$

The method of solution is easily implemented on the computer, and numerical results obtained by $N=8$ in (3.12) and $m=5$ in (3.13). Now we can compare the exact solution with numerical solution. For $t=0.10$ and $x \in[0.10,0.90]$, we calculate $v$ numerically using the proposed method of solution and compare it with the exact solution as in Table 1.

The relative error computed by the formula $\frac{v \text { numerical }-v \text { exact }}{v \text { exact }}$.

\begin{tabular}{|l|l|l|l|l|l|}
\hline$x$ & 0.10 & 0.30 & 0.50 & 0.70 & 0.90 \\
\hline v exact & 0.0898817 & 0.2660619 & 0.4316350 & 0.5800001 & 0.7052425 \\
\hline v numerical & 0.0898818 & 0.2660623 & 0.4316355 & 0.5800058 & 0.7052395 \\
\hline relativ error & $-0,0000058$ & 0,0000017 & 0,0000012 & 0,0000099 & $-0,0000043$ \\
\hline
\end{tabular}

Table1

\section{Uniqueness and Continuous Dependence of the Solution.}

First we establish a priori estimate, then the uniqueness and continuous dependence of the solution with respect to the given data are immediately obtained.

5.1. Theorem. If $u(x, t)$ is a solution of the Problem $(2.3)-(2.4)$, then we have the following inequalities

$$
\begin{aligned}
& \|u(., \tau)\|_{L^{2}(0,1)}^{2} \leq c_{1}\left(\|\varphi\|_{L^{2}(0,1)}^{2}\right) \text { and } \\
& \left\|\frac{\partial u(., \tau)}{\partial t}\right\|_{L^{2}\left(0, T ; B_{2}^{1}(0,1)\right)}^{2} \leq c_{2}\left(\|\varphi\|_{L^{2}(0,1)}^{2}\right),
\end{aligned}
$$

where $c_{1}=\exp \left(a_{0} T\right), c_{2}=\frac{\exp \left(a_{0} T\right)}{1-a_{0}}, 1<a(x, t)<a_{0}$, and $0 \leq \tau \leq T$. 
Proof. If we take the scalar product of the both side of equation (2.3) by $u$, and integrate over $(0, \tau)$, then we have

$$
\begin{aligned}
& \int_{0}^{\tau}\left(\frac{\partial u(., t)}{\partial t}, u\right)_{B_{2}^{1}(0,1)} d t- \\
& \int_{0}^{\tau}\left(\frac{\partial^{2} u(., t)}{\partial x^{2}}, u\right)_{B_{2}^{1}(0,1)} d t \\
= & \int_{0}^{\tau}\left(\int_{0}^{t} a(t-s) u(x, s) d s, \frac{\partial u(., t)}{\partial t}\right)_{B_{2}^{1}(0,1)} d t .
\end{aligned}
$$

Integrating by parts on the left-hand side of (5.2) we obtain

$$
\begin{aligned}
& \frac{1}{2}\left\|\frac{\partial u(., t)}{\partial t}\right\|_{B_{2}^{1}(0,1)}^{2}+ \\
& \frac{1}{2}\|u(., \tau)\|_{L^{2}(0,1)}^{2}-\frac{1}{2}\|\varphi\|_{L^{2}(0,1)}^{2} \\
= & \int_{0}^{\tau}\left(\int_{0}^{t} a(t-s) u(x, s) d s, \frac{\partial u(., t)}{\partial t}\right)_{B_{2}^{1}(0,1)} d t .
\end{aligned}
$$

By the Cauchy inequality, the right-hand side of (5.3) is bounded by

$$
\frac{a_{0}}{2} \int_{0}^{t}\|u(x, s)\|_{L^{2}\left(0, T ; B_{2}^{1}(0,1)\right)}^{2} d s+\frac{a_{0}}{2}\left\|\frac{\partial u(., t)}{\partial t}\right\|_{L^{2}\left(0, T ; B_{2}^{1}(0,1)\right)}^{2} .
$$

Substitution of (5.4) into (5.3) yields

$$
\begin{gathered}
\left(1-a_{0}\right)\left\|\frac{\partial u(., t)}{\partial t}\right\|_{L^{2}\left(0, T ; B_{2}^{1}(0,1)\right)}^{2}+\|u(., \tau)\|_{L^{2}(0,1)}^{2} \leq \\
\|\varphi\|_{L^{2}(0,1)}^{2}+ \\
\frac{a_{0}}{2} \int_{0}^{t}\|u(x, s)\|_{L^{2}\left(0, T ; B_{2}^{1}(0,1)\right)}^{2} d s .
\end{gathered}
$$

By the Gronwall Lemma we have

$$
\begin{aligned}
& \left(1-a_{0}\right)\left\|\frac{\partial u(., t)}{\partial t}\right\|_{L^{2}\left(0, T ; B_{2}^{1}(0,1)\right)}^{2}+\|u(., \tau)\|_{L^{2}(0,1)}^{2} \\
\leq & \exp \left(a_{0} T\right)\left(\|\varphi\|_{L^{2}(0,1)}^{2}\right) .
\end{aligned}
$$

From (5.6), we obtain the estimates (5.1).

5.2. Corollary. If Problem (2.3) - (2.4) has a solution, then this solution is unique and depends continuously on $\varphi$.

\section{References}

[1] M. Abramowitz and I. A. Stegun, Hand book of Mathematical Functions, Dover, New York, 1972.

[2] W. T. Ang, A Method of Solution for the One-Dimentional Heat Equation Subject to Nonlocal Conditions, Southeast Asian Bulletin of Mathematics 26 (2002), 185-191. 
[3] A. Ashyralyev and F.S.O. Tetikoglu, FDM for elliptic equations with Bitsadze-SamarskiiDirichlet conditions, Abstract \& Applied Analysis, 1-22, 2013. Article ID 454831.

[4] A. Ashyralyev and O. Yildirim, A note on the second order of accuracy stable difference schemes for the nonlocal boundary value hyperbolic problem, Abstract \& Applied Analysis, pages 1 - 29, 2012. Article ID 846582.

[5] G. Avalishvili, M. Avalishvili, and D. Gordeziani, On integral nonlocal boundary value problems for some partial differential equations, Bulletion of the Georegian National Academy of Sciences, 5(1):31-37, 2011.

[6] G. Avalishvili, M. Avalishvili, and D. Gordeziani, On a nonlocal problem with integral boundary conditions for a multidimensional elliptic equation, Applied Mathematics Letters, 24(4):566-571, 2011.

[7] S. A. Beïlin, Existence of solutions for one-dimentional wave nonlocal conditions, Electron. J. Differential Equations 2001 (2001), no. 76, 1-8.

[8] G. Berikelashvili and N. Khomeriki, On the convergence of difference schemes for one nonlocal boundary-value problem, Lithuanian Mathematical Journal, 52(4):353-362, 2012.

[9] G. Berikelashvili and N. Khomeriki, On a numerical solution of one nonlocal boundaryvalue problem with mixed Dirichlet-Neumann conditions, Lithuanian Mathematical Journal, 53(4):367-380, 2013.

[10] A. Bouziani, Problèmes mixtes avec conditions intégrales pour quelques équations aux dérivées partielles, Ph.D. thesis, Constantine University, (1996).

[11] A. Bouziani, Mixed problem with boundary integral conditions for a certain parabolic equation, J. Appl. Math. Stochastic Anal. 09 ,no. 3, 323-330 (1996).

[12] A. Bouziani, Solution forte d'un problème mixte avec une condition non locale pour une classe d'équations hyperboliques [Strong solution of a mixed problem with a nonlocal condition for a class of hyperbolic equations], Acad. Roy. Belg. Bull. Cl. Sci. 8 , 53-70 (1997).

[13] A. Bouziani, Strong solution to an hyperbolic evolution problem with nonlocal boundary conditions, Maghreb Math. Rev., 9 , no. 1-2, 71-84 (2000).

[14] A. Bouziani, On the quasi static flexur of thermoelastic rod, Communication in Applied Analysis for Theory and Application, Vol. 6, no.4,pp. 549-568, (2002).

[15] A. Bouziani, Initial-boundary value problem with nonlocal condition for a viscosity equation, Int. J. Math. \& Math. Sci. 30 , no. 6, 327-338.(2002).

[16] A. Bouziani, On the solvabiliy of parabolic and hyperbolic problems with a boundary integral condition, Internat. J. Math. \& Math. Sci., 31, 435-447.(2002).

[17] A. Bouziani, On a class of nonclassical hyperbolic equations with nonlocal conditions, J. Appl. Math. Stochastic Anal. 15 ,no. 2, 136-153.(2002)

[18] A. Bouziani, Mixed problem with only integral boundary conditions for an hyperbolic equation, Internat. J. Math. \& Math. Sci., 26, 1279-1291 (2004).

[19] A. Bouziani and N. Benouar, Problème mixte avec conditions intégrales pour une classe d'équations hyperboliques, Bull. Belg. Math. Soc. 3 , 137-145 (1996).

[20] A. Bouziani and N. Benouar, Sur un problème mixte avec uniquement des conditions aux limites intégrales pour une classe d'équations paraboliques, Maghreb Mathematical Review, vol. 9, no. 1-2, pp. 55-70, (2000).

[21] A. Bouziani and R. Mechri, The Rothe Method to a Parabolic Integro-differential Equation with a Nonclassical Boundary Conditions,Int. Jour. of Stochastic Analysis, Article ID 519684/ 16 page, doi: 10.1155/519684/( 2010).

[22] D. P. Graver, Observing stochastic processes and aproximate transform inversion, Oper. Res. 14, 444-459.(1966).

[23] D. G. Gordeziani and G. A. Avalishvili, Solution of nonlocal problems for one-dimensional oscillations of a medium, Mat. Model. 12, no. 1, 94-103 (2000).

[24] H. Hassanzadeh and M. Pooladi-Darvish, Comparision of different numerical Laplace inversion methods for engineering applications, Appl. Math. Comp. 189 1966-1981(2007).

[25] J.Kacŭr, Method of Rothe in Evolution Equations, Teubner-Texte zur Mathematik, vol. 80, BSB B. G. Teubner Verlagsgesellschaft, Leipzig,(1985).

[26] S.Mesloub and A. Bouziani, On a class of singular hyperbolic equation with a weighted integral condition, Int. J. Math. Math. Sci. 22 , no. 3, 511-519 (1999). 
[27] S.Mesloub and A. Bouziani, Mixed problem with integral conditions for a certain class of hyperbolic equations, Journal of Applied Mathematics, Vol. 1, no. 3, 107-116(2001).

[28] N. Merazga and A. Bouziani, Rothe time-discretization method for a nonlocal problem arising in thermoelasticity, Journal of Applied Mathematics and Stochastic Analysis, Vol. 2005, no. 1 , pp. $13-28,(2005)$.

[29] A. Merad, Adomian Decomposition Method for Solution of Parabolic Equation to Nonlocal Conditions, Int. Journal of Contemp. Math. Sciences, Vol 6, no. 29-32, 1491-1496(2011).

[30] A. Merad and A. L. Marhoune, Strong Solution for a High Order Boundary Value Problem with Integral Condition, Turk. Jour. Math., Vol. 37, no.3, 1-9(2013).

[31] L. S. Pul'kina, A non-local problem with integral conditions for hyperbolic equations, Electron. J. Differential Equations 1999 , no. 45, 1-6(1999).

[32] L. S. Pul'kina, On the solvability in L2 of a nonlocal problem with integral conditions for a hyperbolic equation, Differ. Equ. 36 , no. 2, 316-318.(2000)

[33] L. S. Pul'kina, A mixed problem with integral condition for the hyperbolic equation, Matematicheskie Zametki, vol. 74, no. 3, , pp. 435-445.(2003).

[34] H. Stehfest, Numerical Inversion of the Laplace Transform, Comm. ACM 13, 47-49.(1970)

[35] A. D. Shruti, Numerical Solution for Nonlocal Sobolev-type Differential Equations, Electronic Journal of Differential Equations, Conf. 19 , pp. 75-83.(2010) 
